Cinémas

Revue d'études cinématographiques

Journal of Film Studies

\title{
Certains paysages d'Herzog sous la loupe du système des attractions
}

\section{Viva Paci}

Volume 12, numéro 1, automne 2001

Le Paysage au cinéma

URI : https://id.erudit.org/iderudit/024869ar

DOI : https://doi.org/10.7202/024869ar

Aller au sommaire du numéro

Éditeur(s)

Cinémas

ISSN

1181-6945 (imprimé)

1705-6500 (numérique)

Découvrir la revue

Citer cet article

Paci, V. (2001). Certains paysages d'Herzog sous la loupe du système des attractions. Cinémas, 12(1), 87-104. https://doi.org/10.7202/024869ar
Résumé de l'article

Ce texte présente un parcours de l'évolution du concept d'attraction dans les théories du cinéma. Les lectures qu'en font Eisenstein et Gunning permettent à l'auteur de faire un parallèle avec un autre concept, celui de défamiliarisation. À la lumière de cette mise au point, il devient possible d'établir que la manière dont Herzog utilise les paysages dans des films comme Fata Morgana (1968-1970) et Aguirre, la colère de Dieu (1972) s'inscrit parfaitement dans la préoccupation d'une communication directe comme celle qui entoure le concept d'attraction. L'idée de sublime s'insère dans ce parcours pour souligner le caractère fortement émotif sur lequel jouent les représentations de paysages chez Herzog. 


\title{
Certains paysages d'Herzog sous la loupe du système des attractions
}

\section{Viva Paci}

\begin{abstract}
RÉSUMÉ
Ce texte présente un parcours de l'évolution du concept d'attraction dans les théories du cinéma. Les lectures qu'en font Eisenstein et Gunning permettent à l'auteur de faire un parallèle avec un autre concept, celui de défamiliarisation. À la lumière de cette mise au point, il devient possible d'établir que la manière dont Herzog utilise les paysages dans des films comme Fata Morgana (1968-1970) et Aguirre, la colère de Dieu (1972) s'inscrit parfaitement dans la préoccupation d'une communication directe comme celle qui entoure le concept d'attraction. Lidée de sublime s'insère dans ce parcours pour souligner le caractère fortement émotif sur lequel jouent les représentations de paysages chez Herzog.
\end{abstract}

\section{ABSTRACT}

This article surveys the evolution of the concept of attraction in film theory. Using the readings by Eisenstein and Gunning, in particular, the author proposes a parallel with another concept, that of defamiliarisation. In this light, the use of landscape in films such as Herzog's Fata Morgana (1968-70) and Aguirre, the Wrath of God (1972) responds precisely to the concern for authentic communication underlying the idea of attraction. The concept of the sublime is also considered here, as a way of emphasising the highly emotional nature of the representation of landscape in Herzog. 
Réactivé dans le cadre des études sur le cinéma des premiers temps, le concept d'attraction soulève la question du fonctionnement de la narration cinématographique. Le dénominateur commun des définitions de l'attraction, considérée comme un frein dans l'avancée du récit, renvoie, dans la théorie du cinéma, à ces moments de pause dont la fonction est d'interpeller le spectateur et de lui offrir un moment de spectacle pur. Ce concept est devenu la base d'une lecture du cinéma qui s'oppose, sans toutefois la contredire, à la lecture articulée sur le concept de narration '.

Je traiterai ici de l'attraction ${ }^{2}$ au cinéma en utilisant les définitions formulées par les théoriciens. Dans la Russie des années vingt, ce concept connaît un moment de gloire grâce aux théories d'Eisenstein; en réalité l'idée d'attraction (déjà dominante dans le discours sur le spectacle avant la fameuse projection publique du cinématographe, le 28 décembre 1895 au Salon indien à Paris) fait partie du discours cinématographique depuis les premiers balbutiements de la réflexion sur le cinéma : parfois en opposition, parfois parallèlement à l'idée qui dominait le discours sur le cinéma, à savoir l'idée de pure reproduction, et par la suite, celle de narrativité. Il s'agira donc de mettre en interaction les différentes lectures des attractions au cinéma: certaines synchroniques, d'autres diachroniques puisque ce concept, comme il a été mentionné ci-dessus, fut exhumé pour étudier notamment la période du cinéma des premiers temps. À partir de cette mise au point historique, je procéderai à une nouvelle lecture de films appartenant, eux, au cinéma moderne ${ }^{3}$, et qui semblent se prêter particulièrement bien à la valorisation de l'attraction.

L'attraction [...] est tout moment agressif au théâtre, c'est-à-dire tout élément qui exerce un effet sensoriel et psychologique sur le spectateur, un effet qui doit être vérifié expérimentalement et calculé mathématiquement, aux fins de produire des chocs émotionnels bien précis, qui à leur tour établissent, pour celui qui regarde le spectacle, la condition essentielle de la perception des idéaux et des fins du spectacle (Eisenstein, 1975 , p. 132 - ma traduction). 
En suivant cette voie, Eisenstein affirme que l'objectif de tout théâtre utilitariste est de modeler le spectateur en prédisposant son âme de la façon désirée. Le résultat sera un théâtre loin de la figuration illusoire et de la représentativité : au lieu de construire une illusion dé réalité autosuffisante, la mise en scène théâtrale s'articulera sur un montage d'attractions. Ainsi, dans les années vingt, Eisenstein propose une définition novatrice du concept d'attraction; un concept prêt à devenir la base d'un genre de spectacle qui plonge ses racines dans une volonté de tenir son public, déjà répandue depuis plusieurs décennies, bien qu'à des finalités tout à fait différentes. En effet, le cirque, la foire, le music-hall, le parc d'amusements ${ }^{4}$, avec leurs numéros, constituent un point de repère important pour le cinéaste soviétique, qui y voit la plus intéressante des conceptions du spectacle ${ }^{5}$. Rappelons qu'au cours des années vingt, la fascination exercée par le cirque et les arts populaires est bien marquée, dans le milieu des avant-gardes et ailleurs. Il s'agit de genres d'amusement qui trouvent leur force dans le fait même qu'ils sont composés de plusieurs numéros et attractions, indépendants, autonomes, et montés en séquence (et qu'ils misent en plus sur la mobilité du spectacle et sur la participation du spectateur).

Le point de vue d'Eisenstein est celui du réalisateur; son texte constitue une sorte "de mode d'emploi » qui s'adresse à la catégorie des réalisateurs-monteurs, ceux qui voient dans le théâtre, dans le cinéma un possible instrument d'autorévélation idéologique pour un public prolétarien: "Les réflexions qui vont suivre ne s'affichent pas comme manifeste ou déclaration programmatique, elles sont une tentative pour s'orienter dans les principes de notre difficile métier" (Eisenstein, 1986, p. 227 ma traduction). L'objet central de sa recherche est le spectateur. Le théâtre, le cinéma et l'art en général doivent être utiles, c'està-dire agir sur le spectateur, stimuler sa réflexion sur sa propre condition. L'attraction sera le moyen idéologique privilégié pour secouer le spectateur et par là même le faire réfléchir. Le spectacle doit être un montage d'attractions reliées les unes aux autres de façon à ce que chacune provoque une réaction émotive: le résultat sera la compréhension profonde du message de l'auteur. La définition de l'attraction rapportée plus haut se réfere au 
théâtre, mais Eisenstein trouvera dans le cinéma, deux années plus tard, le moyen le plus approprié pour travailler les attractions: le montage ${ }^{6}$.

Il faut rappeler que le cinéma, ou mieux, le cinématographe ${ }^{7}$, jusqu’à quelques années auparavant, était considéré lui-même comme une attraction, et présenté comme telle à l'intérieur d'autres institutions de spectacle, comme le music-hall, la foire, le parc d'amusement ${ }^{8}$. Le cinéma constitue donc, pour Eisenstein, l'unique forme en mesure d'exprimer pleinement son «montage des attractions». Car le cinéma est dans son essence même montage, il l'est dans son dispositif, il l'est dans la tête, dans les projets, dans la façon de voir du réalisateur. Si, en effet, pour une certaine école de pensée, le cinéma implique toujours et quand même une narration ", ici, dans l'optique eisenstenienne, le cinéma, par ses propres caractéristiques structurelles, s'apparente au montage: montage dans la pellicule puisqu'elle est inévitablement assemblage et juxtaposition de photogrammes; montage comme parcellisation de la réalité créée par l'œil de la caméra, incapable, par sa nature, d'enregistrer la réalité de façon intégrale, et aussi parcellisation voulue par le projet du réalisateur. Le photogramme restitue la réalité captée dans le cadre, le cadre restitue ce que l'œil de la caméra arrive à capter dans son champ, et le plan isole ce que le réalisateur voit dans la réalité. Le cinéma est montage, car il crée et accumule des associations nécessaires dans la psyché du spectateur "en les faisant naître des éléments singuliers d'un fait découpé, pratiquement, en segments de montage" (Eisenstein, 1986, p. 229 - ma traduction).

L'attraction, on le sait, est ce moment spectaculaire qui interpelle directement le spectateur, en allant presque le chercher dans son fauteuil, pour le pousser à réagir. Mais, si dans l'ambiance des années pivots, comme les années vingt en Russie, interpeller le spectateur veut dire le pousser à prendre part aux dynamiques sociales et politiques et l'exhorter à agir ${ }^{10}$, le fait de se servir d'un moment énergétique du spectacle, dans un but précis, est commun à plusieurs créations artistiques. Une attraction, disait-on avec Eisenstein, est ce moment agressif du théâtre, c'est-à-dire ce moment où chaque élément tend à exercer un 
effet sensoriel et psychologique puissant sur le spectateur. Une sorte de choc nerveux qui, comme s'il permettait la sécrétion d'une grande quantité d'adrénaline dans l'organisme du spectateur, le rend beaucoup plus réceptif que d'habitude, plus propice à la communication, et donc à la transmission de l'idéologie portée par le spectacle ${ }^{11}$.

L'attraction casse l'automatisme de la perception (au théâtre et au cinéma) et permet à la réalité représentée dans le spectacle de percer le dispositif de la fiction. Ainsi l'idéologie sous-jacente peut être dévoilée. L'effet sensoriel casse les automatismes du spectacle d'amusement bourgeois éloigné de la réalité (tout comme, dans l'univers chklovskien, le langage poétique permet aux mots d'accomplir leur vraie tâche, celle de communiquer le monde). Une acrobatie exécutée par un acteur sur la scène, ou le très gros plan d'une image qui n'a pas de rapport de cause à effet avec les images contiguës, permet donc à la vérité (l'idéologie nécessaire pour l'Union soviétique selon Eisenstein) de percer l'artifice scénique et de paraître dans toute son épiphanie. Casser l'identification, bourgeoise, par le biais d'une attraction, casser la narration linéaire, et rendre visible la construction, les procédés du théâtre par exemple, équivaut à rendre opaque le médium thêâtre, à le rendre résistant à l'identification ${ }^{12}$, et en revanche, par là même, transparent à l'idéologie soutenue. En fait, on pourrait considérer que l'attraction est au cinéma ce que la singularisation est à la poésie, et par là-même au langage. De plus, l'effet sensoriel devait être vérifié expérimentalement et calculé mathématiquement, de façon à pouvoir prévoir la production des charges émotives, lesquelles, disposées dans une certaine structure significative - grâce au montage - , donnent accès, de façon scientifique, au contenu idéologique du spectacle. À travers une série de stimuli, d'impulsions et de contreimpulsions, le montage est investi du pouvoir de modeler la conscience du spectateur pour faciliter la réception du contenu global de l'œuvre, c'est-à-dire l'adhésion totale au message idéologique. Or, une caractéristique commune à de nombreuses créations artistiques, au-delà des époques, est justement d'exploiter des moments particulièrement forts de la représentation pour en faire, comme dans le cas des attractions eisensteiniennes, un 
canal de communication privilégié entre le programme de l'auteur-réalisateur (ou réalisateur-monteur, pour le dire comme Eisenstein) et le spectateur.

Werner Herzog est, à ce propos, un réalisateur qui représente de façon emblématique, selon des modalités différentes évidemment, cette tension entre auteur et spectateur que seul peut offrir l'usage d'images fort intenses, comme justement celles qu'Eisenstein appelait des attractions. Mais avant de passer à l'observation de quelques-uns de ses films, et pour fournir d'autres repères sur la question des attractions au cinéma, il nous faut présenter un autre discours majeur de la théorie du cinéma portant sur les attractions, mais qui est de nature différente de celui d'Eisenstein.

Dans les années quatre-vingt, le mot attraction est repris par certains des nouveaux historiens ${ }^{13}$. Je pense notamment à André Gaudreault, Rick Altman, Charles Musser et plus particulièrement à Tom Gunning, qui a proposé plusieurs textes sur la question. À remarquer que Gunning ne se pose aucun but pratique en parlant d'attraction comme le faisait Eisenstein; pour l'historien, il s'agit de trouver dans le concept d'attraction un point de vue d'où regarder avec des yeux différents de ceux des historiens qui l'ont précédé, la façon de faire du cinéma qui existait pendant les premières décennies d'usage du cinématographe. Ce regard historique reconsidère le cinéma des premiers temps, et plus généralement toute la culture du spectacle qui chevauchait les XIX et $X^{e}$ siècles, à travers le concept d'attraction. Après le colloque de Brighton en 1978, et les intuitions de quelques chercheurs (comme Noël Burch, et Gunning luimême), on commença à ne plus regarder le cinéma des origines comme un noyau enveloppant les germes du cinéma futur, germes encore insuffisamment développés en raison des limites toutes techniques des appareils de l'époque. Le renouveau du regard des historiens a permis de voir ce cinéma-là comme quelque chose de différent: un cinéma autre que celui que l'on connaîtra à partir de 1910, avec une autre façon d'être montré et une autre façon d'être regardé.

Dans ses textes, rédigés entre 1986 et 1993, Tom Gunning parle d'attraction dans le cinéma des premiers temps après con- 
sidération de ses mécanismes et de son action sur le spectateur. Il distingue la "narration" mise en place par ce cinéma de celle du cinéma narratif, ainsi nommé d'après une théorie narrative classique. Il abandonne d'abord la théorie traditionnelle (évolutionniste) de l'histoire du cinéma, qui cherche les traces d'une continuité dans l'histoire du film; il affirme ne pas accepter ce que le "regard narratif " implique, à savoir : considérer le cinéma uniquement à partir de sa capacité à raconter une histoire et évaluer les cinéastes d'après leur contribution au développement de cette aptitude. Le cheminement de Gunning n'exclut pas de façon catégorique tout aspect narratif, et il articule plusieurs raisonnements à partir de l'idée de continuité narrative, même si ce n'est pas sur le degré de complexité narrative que reposent ses classifications. Son attention, ainsi que le concept même d'attraction - développé avec Gaudreault ${ }^{14}$ —, se concentrent, avec quelques variations de classification, sur la période couvrant les années 1895/1910. Selon Gunning, le cinéma de cette période, totalement différent de celui des périodes suivantes, enfonce ses racines dans un système de spectacle qu'il définit comme système des attractions ${ }^{15}$. Un tel système existait déjà avant la naissance du cinématographe, dans la culture populaire du XIX ${ }^{c}$ siècle, quand la constante évolution des moyens techniques donnait au spectacle l'attrait de leur nouveauté; le cinématographe, inséré à juste titre dans cette dynamique, représente la dernière nouveauté, celle qui a le plus de pouvoir d'attirer le public, grâce au réalisme saisissant des prises de vues animées. Le cinématographe, attraction par lui-même, est donc présenté comme telle dans les foires et dans les parcs d'amusement (parc d'amusement ou bien... d'attractions! comme on le dit communément), machine magique en mesure de reproduire la réalité et d'attirer un grand nombre de spectateurs.

En comprenant ces premières données de base, en essayant de saisir l'approche de ceux qui voyaient et faisaient le cinéma pour la première fois, on comprend mieux qu'une pensée soumise à l'hégémonie narrative du cinéma est en porte-à-faux quand il s'agit d'analyser le cinéma des origines. Ce cinéma-là n'avait pas la narration comme but; son but, le but original était de montrer. D'abord montrer la nouvelle façon de voir à travers l'œil de 
la caméra, et ensuite montrer de nouvelles images, exotiques, insolites et même fantastiques, dans un parcours qui ne sépare pas Méliès de Lumière. Montrer l'attraction, voilà la pulsion que Gunning reconnaît dans le cinéma des origines, qu’il définit pour cette raison "cinéma des attractions". Cela ne signifie pas que, selon Gunning, il n'existe pas de narration dans le cinéma des attractions. En effet, même les films composés d'un seul plan sont des cellules narratives, comme l'observe pour sa part Gaudreault (voir plus haut), qui souligne que la narration est implicite au cinéma parce que celui-ci est en mesure d'enregistrer le passage du temps et conséquemment les changements impliqués par ce passage. Dans la lecture de Gunning, toute coupure nette est stérile: il ne faut pas croire que le cinéma des attractions disparaît avec l'institutionnalisation du cinéma narratif, mais plutôt qu'il s'y intègre (comme nous l'enseigne Star Wars).

Comme ce sera le cas pour Eisenstein, l'attraction dans le cinéma des premiers temps doit toucher le spectateur, avec la différence que le réalisateur soviétique veut éduquer le spectateur, en le choquant, alors que le but de ceux qui faisaient du cinéma, dans les premiers temps, était au contraire de l'attirer en le séduisant (par l'attraction), pour l'inciter à acheter un billet. Le cinéma des attractions est manifestement conscient de la présence du spectateur, il a une attitude exhibitionniste (pensons, comme nous le rappelle Gunning, aux nombreux regards des magiciens adressés à la caméra, chez Méliès par exemple) qui s'oppose au cinéma narratif dont l'ambition est d'offrir au spectateur une place rassurante d'où regarder sans être dérangé. Le cinéma narratif ne parle pas directement au spectateur, il est concentré sur lui-même, sur l'évolution de l'histoire. Baudrillard parlait du plaisir qu'on éprouve quand on peut tout voir sans être présent, tout comme Dieu, qui n'existe pas et qui peut regarder le monde depuis son absence! Selon Gunning, le rapport que le cinéma des attractions instaure avec le spectateur a pour fonction d'expliciter, à l'intérieur même du film, une ou plusieurs instances monstratives: le showman (encore la figure du magicien comme dans "Now you see it, now you don't"), les nombreux regards des acteurs à la caméra, mais aussi certaines 
instances extérieures au film comme le bonimenteur, ou encore certains titres qui expliquent de façon très détaillée l'attraction présentée. S'adresser directement au spectateur en lui annonçant l'attraction, c'est une stratégie visant à créer de l'attente pour que le plaisir soit à son comble lors du numéro sensationnel. Lattraction en elle-même ne dure qu'un moment, puis elle disparaît: elle dure le temps nécessaire pour être perçue (si son exposition se prolongeait indûment, elle perdrait le pouvoir d'attirer, alors que, au contraire, en la faisant disparaître au plus vite, on est assuré d'atteindre le spectateur — toujours cette même histoire de l'attrait du jouet tout neuf...).

Lattraction, telle que formulée par Gunning dans le système des attractions monstratives à propos du cinéma des premiers temps, est en même temps climax et dénouement; le plaisir du spectateur est tiraillé entre l'excitation de l'attraction et la frustration engendrée par sa disparition. Le temps de l'attraction est celui du pur moment présent - le moment de son apparition -, il n'offre pas de continuité, mais se fait au contraire interruption, temporalité brisée, ou encore temporalité nouvelle qui s'instaure entre un spectateur impressionné et les coups instantanés que le cinéma lui assène.

Le cinéma des origines, comme Gunning le présente, est une sorte de cinéma pur ${ }^{16}$, au sens où il ne peut compter que sur des images animées, ces composantes du langage cinématographique qui lui sont exclusives, et où aucune échappée par la narration ne lui est permise. Depuis les origines du cinématographe, plusieurs parcours ont été tracés qui ont quelque chose à voir avec l'aventure du regard, où la réalité réussit à imposer ses propres directions, souvent imprévisibles. Qu'il s'agisse du cinéma à vocation documentaire ou du cinéma à vocation scientifique, il permet de faire revoir, et donc de faire mieux comprendre, ce qui n'avait été vu qu'une seule fois, ce qui avait été mal vu, ou pas vu du tout ${ }^{17}$.

En se dédiant à la recherche d'un cinéma pur (selon ses intentions autoproclamées), Herzog a créé un cinéma des attractions (bien que certaines différences soient notables entre "ses" attractions et celles qui sont venues avant lui). Werner Herzog, qui n'a été l'élève d'aucune école de cinéma et que l'on peut 
rapprocher de cette idée romantique du "mono-maniaque inconsidéré" que Bazin défendait dans Le Mythe du cinéma total (1958) a un rapport très personnel avec la pratique cinématographique $^{18}$. Comme s'il était toujours en train de chercher la façon la plus appropriée de communiquer et de chercher les images qui expriment, le plus adéquatement possible, la réalité (démarche chklovskienne...). Le monde dans lequel on vit est fait d'images, mais ce sont toujours les mêmes, éternellement reproductibles et continuellement remises sur le tapis, des images publicitaires, télévisuelles qui ne sont plus des représentations du vrai, mais des reprises d'images précédentes désormais codifiées. Et ce monde d'images a développé nos capacités visuelles et a fait surgir le besoin de nouvelles stimulations, tout en nous accoutumant à elles. Ainsi Herzog est-il continuellement à la recherche de la condition qui était le propre du cinéma des origines, quand toutes les images représentaient une nouveauté. Aujourd'hui, il doit cependant aller chercher ces images nouvelles dans des lieux insolites, exotiques, là où tout n'a pas encore été exploré par le regard enregistreur de l'homme: en Afrique, en Amérique du Sud, dans des îles désertes, des lieux abandonnés par les hommes ${ }^{19}$ (notons qu'Herzog porte aussi son attention sur les hommes qui sont des exclus de la société et qui témoignent d'une réalité différente, plus profonde). L'idée d'Herzog se fonde sur une conception assez simple: le cinéma est le langage le plus en mesure d'aller au-delà de la réalité, justement parce qu'il est la façon de la reproduire le plus fidèlement.

À la base de son cinéma, il y a la volonté de secouer le spectateur avec des images insolites et pures puisque non encore infectées pas le virus de la répétitivité ${ }^{20}$, et ces images sont, c'est là notre propos, des attractions, celles-là mêmes que théorisait Eisenstein. Les deux réalisateurs ont en commun le but d'éduquer le spectateur, même si la leçon d'Herzog porte sur la façon de regarder, et non sur le contenu. Le type de regard que Herzog veut que le spectateur adopte lui sera nécessaire pour aller au fond des choses, pour scruter ce qui se trouve derrière l'apparence. En poursuivant cette comparaison entre le système des attractions - ainsi qu'il a été formulé à partir d'Eisenstein et de Gunning - et l'attraction telle qu'on la voit chez Herzog, un 
autre aspect, celui du temps de l'attraction, se signale à notre attention. Dans le cinéma d'Herzog, la notion de temporalité de l'attraction, celle de "Now you see it, now you don't ", disparaît. Les images-attractions d'Herzog sont souvent répétées et le regard s'y arrête longuement. Grâce à d'interminables plansséquences, Herzog explore les paysages sans craindre la monotonie comme l'aurait craint le cinéma classique; il vise à ouvrir le regard vers des dimensions plus vastes. Le paysage: donnée fondamentale.

Herzog introduit le paysage comme attraction et le fait entrer dans la dimension du sublime. Tout comme le sublime défini par Kant, les paysages trouvent leur finalité dans le rapport qu'ils instaurent avec notre esprit et, tout comme le sublime, peu importent qu'ils soient magnifiques, grandioses ou horribles et écrasants, pourvu qu'ils émeuvent (tandis que le beau charme). Cette forte émotion s'adresse directement à la raison, pour la stimuler, pour la faire réfléchir sur elle-même et sur ses propres capacités. Lémotion véhiculée par le sublime génère un retour réflexif de l'esprit humain sur lui-même; c'est vers ce but que tend la recherche de paysages extrêmes chez Herzog. Il lui faut travailler sur le sublime du paysage, sur cette attraction, pour faire travailler le spectateur qui, dans la vision d'Herzog, risque l'accoutumance devant le mimétisme et la représentativité de belles (au sens kantien) images, affectées par le virus de la répétitivité, comme je le disais plus haut. Herzog affirme que ses films naissent souvent d'un paysage qui stimule ses rêves éveillés; le paysage est l'attraction constante de ses films; les procédés de monstration qui le visent nous font ressentir la puissance attractive qui l'habite. Aguirre, la colère de Dieu (1972), Fata Morgana (1968-70), et bien d'autres aussi, sont de ces œuvres où. le paysage se fait définitivement protagoniste. Dans Aguirre, le paysage est le moteur de l'action depuis le premier plan. Le fleuve tout d'abord, qui remplit l'écran juste après le générique, sera le vrai maître de toute la deuxième partie du film. Puis, après un panoramique aérien qui nous fait percer les nuages, les Andes apparaissent, et lentement nous nous approchons d'un des nombreux versants du massif et apercevons une ligne en mouvement. Nous nous approchons encore un peu 
plus pour finalement découvrir qu'il s'agit d'une colonne d'hommes et d'animaux. Dès le début, la présence du paysage est envahissante et rend l'homme infiniment petit et inadéquat (comme cela sera démontré diégétiquement quand l'expédition sera obligée de s'arrêter, embourbée dans le labyrinthe dense et incompréhensible de la jungle). Le fleuve, lent, porte les radeaux vers une direction qui se révèle sans but; la nature est toujours indifférente à l'homme, elle ne lui donne aucun signe de réconfort, elle se moque même de lui, comme lorsqu'un navire apparaît soudain, au sommet des arbres, devant le regard abasourdi des hommes du radeau. Les premières séquences semblent illustrer l'une des définitions kantiennes du sublime, une définition qui nous permet de jeter un pont entre paysage et attraction:

L'aspect d'une chaîne de montagnes dont les sommets couverts de neige s'élèvent au-dessus des nuages, la description d'un violent orage, ou la peinture que nous fait Milton du royaume infernal, excitent en nous une satisfaction mêlée d'horreur (cité dans Khodoss, 1955, p. 40).

Dans Fata Morgana, la nature joue le premier rôle. Quelques êtres humains (des survivants, croit-on) se laissent emporter, à force de confrontation avec elle. C'est l'immense paysage africain qui se montre. "La nature évoque surtout les idées du sublime par le spectacle du chaos, des désordres les plus sauvages et de la dévastation, pourvu qu'elle y manifeste de la grandeur et de la puissance" (p. 43), nous rappelle Kant ${ }^{21}$. Dans un planséquence continu, composé par des mouvements horizontaux très rapides, au sol ou dans les airs, le paysage défile devant nos yeux, sans jamais s'arrêter ${ }^{22}$. Il n'en a pas besoin, car sa beauté et son pouvoir enchanteur suffisent à témoigner du sens de la défaite du genre humain, dont Herzog veut livrer témoignage. Mais les objets et les hommes aussi deviennent ici des attractions: la caméra s'approche lentement d'une épave d'avion, et avec un plan-séquence composé des mouvements circulaires, elle évolue entre les débris pour arriver à une prise de vue complète de l'épave (même mouvement pour nous dévoiler un arbre, par exemple). Ces mouvements tendent à cacher l'objet jusqu'à ce 
que nous soyons prêts à le regarder avec d'autres yeux; l'image de l'objet qui nous était familier, ainsi présentée, est singularisée, et nous devenons capables de comprendre à quoi elle renvoie vraiment, sans automatisme. Le regard de la caméra déforme notre façon habituelle de voir, pour nous dépayser, et donc nous toucher, pour enfin nous faire réfléchir. Les hommes sont traités aussi de façon particulière. Tous les personnages que la caméra rencontre sont filmés en plan fixe. Impitoyable, la caméra ne laisse aucune échappatoire à celui qui est filmé pas plus qu’à nous qui regardons le film. Dans le plan américain sur un enfant qui tient un renard dans ses bras, le regard de la caméra le fixe pendant deux minutes, et ces deux minutes deviennent une éternité devant l'impatience du sujet filmé, ainsi obligé de ne pas bouger, et devant notre même impatience, à nous spectateurs qui ne sommes pas habitués à fixer du regard pendant si longtemps (cinéma exhibitionniste, disait-on avec Gunning...). Cette image stimule fortement le spectateur, car elle rompt avec sa façon habituelle et rassurante de voir les choses, sans qu'il ne se fasse interpeller directement par les personnages filmés. Herzog lui-même nous interpelle directement, et nous abandonne devant cette image. Ce regard à la caméra, ce dévoilement de l'artifice de la représentation cinématographique, laisse percevoir la présence du réalisateur, et entrevoir une sorte de chemin préférentiel, direct, vers le spectateur: expliciter l'artifice, la présence de l'auteur et la présence de la caméra pour faire comprendre au spectateur que le réalisateur s'adresse directement à lui. Je parlais plus haut de ce canal de communication privilégié entre l'auteur de l'cuuvre et son spectateur lorsque se présentent des moments forts de la représentation. C'est ici un autre point de communion avec le système des attractions monstratives qui fonctionne grâce, entre autres, à la façon de se référer directement au spectateur en dévoilant, en montrant l'artifice.

Les attractions, qu'elles soient celles du cinéma des premiers temps, d'Eisenstein ou d'Herzog — et j'aurais pu parler aussi des étincelles de photogénie de Jean Epstein, de la cinégraphie de Germaine Dulac, des numéros de la comédie musicale américaine, des effets spéciaux, de l'usage manifeste, tant à la mode, de plusieurs formats de support $(16 \mathrm{~mm}, 35 \mathrm{~mm}$, vidéo, 
numérique), de la caméra à l'épaule, des images sales et du documentaire qui se coltine la réalité sans médiation - nous obligent à faire un effort émotif et intellectuel, sans que l'auteur ou la diégèse n'aient préalablement tout digéré pour nous. Les attractions livrent une expérience filmique du temps discontinu, fondée sur de pures stupéfactions et fascinations visuelles. Il serait ainsi possible à mon avis de travailler sur un corpus hétérogène de films, réunis par l'expérience commune des attractions, créés avec des possibilités cinématographiques différentes, où règne une temporalité disjointe et ponctuelle basée sur la présentation et l'exhibition visuelles, et dont la manière de fasciner le spectateur se démarque fortement des configurations cohérentes de la narration.

On peut retenir de ce qui précède qu'une fois pensée l'attraction, il est possible d'envisager une façon d'être entrainé par un film, qui soit très différente de celle qu'engendre l'absorption diégétique, favorisée par la narration linéaire.

\section{Université de Montréal}

\section{NOTES}

1. "Le système [des] attractions monstratives ne connaît que très faiblement le régime de la narration filmique [...]. Y règne, au contraire, la monstration filmique dont le domaine privilégié et l'unité de base est le plan [...], unité autonome et autarcique" (Gaudreault et Gunning, 1989, p. 57-59). La communication date de 1985. C'est moi qui souligne.

2. J'ai justement coordonné un séminaire au DAMS de l'Université de Bologne, à l'hiver 2000, intitulé Il Cinema: unattrazione. Je tiens à remercier Alberto Gini qui, pendant ses recherches de maîtrise (Il Linguaggio puro della nonfiction), a partagé avec moi des réflexions stimulantes sur le système des attractions dans le cinéma d'Herzog.

3. Je reprends dans ce passage l'expression "cinéma moderne" telle que l'utilise Youssef Ishagpour (1986).

4. Voir à ce sujet l'intérêt de Tom Gunning pour l'institution "fairground". Le cinéma des premiers temps, qui n'était pas encore autosuffisant dans la façon d'exhiber et de raconter, semblait s'appuyer sur la façon de soutenir l'intérêt du public des parcs d'amusement: «[...] viewing experiences related more to the attractions of the fairground than to the traditions of the legitimate theatre. The relation between film and the emergence of the great amusement parks, such as Coney Island, at the turn of the century provides rich ground for rethinking the roots of early cinema " (Gunning, 1990b).

5. "Le music-hall et le cirque sont une école pour le monteur (réalisateur, ndr), car réaliser un bon spectacle (du point de vue formel) signifie préparer un programme de 
music-hall et de cirque, tout en partant de situations prises dans le texte théâtral de base" (Eisenstein, 1975, p. 134). Ma traduction (c'est moi qui souligne).

6. "Lapplication de la méthode du montage des attractions dans le champ cinématographique est encore plus appropriée que dans le domaine du théâtre, puisque cet art, que je définirais "art des combinaisons", en montrant non pas des faits réels mais des reflets (photographiques) conventionnels $[\ldots]$, a besoin, même pour exposer des faits tout simples, de la comparaison (c'est-à-dire d'une présentation séquentielle particulière de ses composantes). Le montage donc [...] est une condition essentielle du cinéma [...]"(Eisenstein, 1986, p. 228-229). Ma traduction.

7. «... le parole sono importanti...": cette pointilleuse affirmation d'un Nanni Moretti puriste de la langue in Palombella rossa (1989) pourrait être la devise d'André Gaudreault (1997) qui, dans son article fondamental intitulé "Les vues cinématographiques selon Georges Méliès, ou : comment Mitry et Sadoul avaient peut-être raison d'avoir tort (même si c'est surtout Deslandes qu'il faut lire et relire)", défend l'idée qu'une mise au point du jargon cinématographique, selon une mémoire historique plus juste, qui restitue des mots aujourd'hui désuets (cinématographiste au lieu d'un mot plus moderne comme cinéaste, par exemple), nous permettrait de mieux comprendre l'univers cinématographique des premières années.

8. "Le cinématographe a été longtemps considéré comme une "attraction". C'était un des numéros de programme dans les cafés-concerts et dans les music-halls, au même titre qu'un chanteur ou qu'un acrobate. [...] Aujourd'hui, il n'en est plus ainsi [...]. Le cinéma n'est plus une attraction, mais un spectade bien classé " (Fouquet, 1912). Cet article m'a été fourni par André Gaudreault, dans le cadre du séminaire "Problèmes et méthodes de l'histoire du cinéma", 1999/2000, à l'Université de Montréal.

9. Voir à ce sujet la position d'André Gaudreault (1999), qui soutient que le cinéma sécrète de la narration; cf. surtout le chapitre intitulé "À la recherche du premier récit filmique ".

10. Cette époque était celle des usines qui se transformaient en thêâtres, des symphonies urbaines pour sirènes et sifflets des grands concerts futuristes, et de la culture au service de l'idéologie; le théâtre et les autres arts étaient instruments d'autorévélation idéologique pour le public prolétaire. C'est en 1924 qu'Eisenstein met en scène, au Proletkult, la pièce "Masques à gaz" de Tretiakov dans une usine. On peut trouver un bon panorama de l'activité théâtrale d'Eisenstein dans "Le Proletkult et Eisenstein" de Bernard Eisenschitz (1970).

11. Il est utile de rappeler l'existence du précieux texte de Viktor Chklovski (1965), «L'art comme procédé». L'idée de Chklovski présentée dans cet article constitue l'origine, dans la Russie prérévolutionnaire, de cetre ligne de pensée dont Eisenstein est un protagoniste de poids.

12. Il est évident qu'on se retrouve ici dans un univers qui est attribué d'habitude aux théories brechtiennes du théâtre épique. Il serait intéressant de parcourir les écrits de Brecht qui théorisent de près l'idée de distanciation, dans la mesure où celle-ci s'apparente au système de Chklovski, et donc aux attractions eisensteniennes. Brecht avait d'ailleurs probablement lu "L'art comme procédé». En contrepartie, le Verfremdung brechtien et l'Ostranïenüe chklovskien ont été par la suite très souvent traduits par le même mot: straniemento en italien et distanciation en français (même si, dans le cas de Chklovski, on retrouve plus souvent: singularisation ou défamiliarisation). Je me limite ici à noter les trois textes de Brecht qu'il est utile de lire dans ce sens: "Ovation pour Shaw" (1963), Petit Organon pour le théatre (1963), et "Notes sur l'opéra" (1979). On peut également y lire les pages particulièrement intéressantes sur l'idée de montage dans le théâtre épique de Brecht, qui se retrouve ainsi dans la lignée d'Eisenstein et dans celle de Walter Benjamin (1969). 
13. On a l'habitude de nommer ainsi une génération d'historiens qui participèrent au fameux congrès de la FIAF tenu à Brighton en 1978, où l'on présenta plus de six cents films de la période 1900-1906, ouvrant ainsi un champ de réflexion plus riche que jamais. Pour un bilan de la nouvelle histoire du cinéma appliquée au cinéma des premiers temps en particulier, cf. André Gaudreault et Denis Simard (1995). Cf. aussi Jacques Aumont et al. (1989).

14. André Gaudreault et Tom Gunning (1989), dans leur communication de 1985.

15. André Gaudreault (1997, p. 129) écrit: "Cinéma des attractions, expression d'une problématique vigoureuse et dynamique, par rapport à cinéma des premiers temps, expression d'une temporalité qui suit platement son cours. Cinéma des attractions, qui a le mérite de raviver un concept qui était en vogue, à l'époque, dans le monde de la cinématographie ainsi que dans une grande variété d'autres séries culturelles, et dont la fortune suit probablement une courbe inversement proportionnelle à l'institutionnalisation du cinéma." "

16. En utilisant un terme comme pur, je ne veux pas pour autant reproduire une thèse inverse et symétrique à la théorie "évolutionniste", qui soutiendrait que le cinéma serait handicapé par le manque de narration. Je ne veux pas non plus présenter une thèse qui témoigne d'une dégradation du cinéma du pur vers l'impur, du système des attractions au cinéma narratif.

17. Une observation de Benjamin me semble bien à-propos: «Par les gros plans tirés de l'inventaire phorographique, par la nouvelle évidence qu'il donne à des détails qui restaient dissimulés dans les accessoires courants de notre vie, par l'exploration de milieux banals sous la conduite géniale de l'objectif, le cinéma nous fait mieux discerner les contraintes qui régissent notre existence, mais il nous ouvre en même temps un espace de jeu, énorme et insoupçonné! Les bars et les rues de nos grandes villes, nos bureaux et nos meubles, nos gares et nos usines semblaient nous emprisonner sans espoir. Le cinéma vint, qui fit sauter ce monde carcéral à la dynamite de ses dixièmes de seconde de telle sorte qu'impassibles, parmi les ruines dispersées sur une vaste étendue, nous entreprendrons d'aventureux voyages. Le gros plan étire l'espace, le ralenti étire le mouvement. Et de même qu'il ne s'agit pas du tout, avec le grossissement, de faire voir clairement ce que nous verrions sans cela confusément, mais bien de faire apparaître des formations structurelles totalement neuves de la matière, le ralenti fait non seulement apparaître des figures bien connues de mouvement, mais découvre encore dans ces figures des figures inconnues [...]" (Benjamin, 1997, p. 5657). Je remercie Pierre-Emmanuel Jaques, de l'Université de Lausanne, d'avoir porté à mon attention cette réflexion benjaminienne, et pour les précieux échanges que j’ai eus avec lui sur le fonctionnement du système des attractions, non seulement dans le cinéma des premiers temps, mais dans le cinéma en général.

18. Toute sa production réfléchit ce qu'on vient de dire, et je donnerais son dernier film, Mon ennemi intime (1999), comme cas exemplaire.

19. Tom Gunning fait l'observation suivante: «Une attraction est un [pur] moment de manifestation visuelle. Elle forme la base de grand nombre de divertissements populaires [...] L'attraction possède un intérêt visuel qui peut découler d'un grand nombre de points. La nouveauté et l'exotisme peuvent la motiver; l'étrange et l'inhabituel en constituer un genre" (Gunning, 1995, p. 179). C'est moi qui souligne.

20. Virus de la répétitivité qui entraîne l'automatisation... Encore une fois Chklovski nous éclaire: "Ainsi la vie disparaît, se transformant en un rien. L'automatisation avale les objets, les habits, les meubles, la femme et la peur de la guerre [...] Et voilà que pour rendre la sensation de la vie, pour sentir les objets, pour éprouver que la pierre est de pierre, il existe ce qu'on l'appelle l'art. Le but de l'art, c'est de donner une sensation de l'objet comme vision et non pas comme reconnaissance [...]" (Chklovski, 1965, p. 83). C'est moi qui souligne. 
21. On peut trouver de la matière pour renforcer cette comparaison entre le sublime kantien et l'attraction selon Eisenstein dans la Critique du jugement: «[...] l'objet [de la nature] est propre à servir de présentation à une sublimité qui peut être trouvée dans l'esprit; car le sublime authentique ne peut se trouver contenu dans aucune forme sensible; il ne concerne que les idées de la raison, qui $[\ldots]$ sont excitées et évoquées dans l'espri: par cette disconvenance même, qui peut se présenter sous forme sensible." Emmanuel Kant, Critique du jugement (dans Khodoss, 1995, p. 42-43). Ce qui explique, une fois de plus, l'attitude d'Herzog qui recherche dans les images d'une nature choquante une sorte de miroir où l'homme peut voir, revoir et travailler son intellect. Dans cette lecture, le sublime et l'attraction sont générés par le rapport entre le paysage filmé par Herzog et l'esprit du spectateur.

22. Cela me fait penser à plusieurs vues exotiques des frères Lumière, comme par exemple Niagara (1897) et Egypte: panoramas des rives du Nil (1896).

\section{RÉFÉRENCES BIBLIOGRAPHIQUES}

Aprà, 1996: Adriano Aprà (dir.), Il Cinema e il suo oltre, Pesaro, Mostra internazionale del nuovo cinema, 1996.

Aprà, 1997 : Adriano Aprà (dir.), Le Avventure della nonfiction. Il cinema e il suo oltre 2, Pesaro, Mostra internazionale del nuovo cinema, 1997.

Aumont, 1979: Jacques Aumont, Montage Eisenstein, Paris, Albatros, 1979.

Aumont et al., 1989: Jacques Aumont, André Gaudreault et Michel Marie (dir.), Histoire du cinéma. Nouvelles approches, Paris, Publications de la Sorbonne, 1989.

Bazin, 1958: André Bazin, "Le mythe du cinéma total", dans Qu'est-ce que le cinéma?, vol. I, Paris, Éditions du Cerf, 1958.

Benjamin, 1969 : Walter Benjamin, Essais sur Bertold Brecht, Paris, Éditions Maspero, 1969.

Benjamin, 1997: Walter Benjamin, "L'œuvre d'art à l'époque de sa reproductibilité technique", Sur l'art et la photographie, Paris, Éditions Carré, 1997.

Brecht, 1963: Bertold Brecht, "Ovation pour Shaw", dans Écrits sur le théâtre, vol. 1, Paris, L'Arche, [1926] 1963.

Brecht, 1963 : Bertold Brecht, Petit Organon pour le théâtre, Paris, LArche, [1949] 1963.

Brecht, 1979: Bertold Brecht, "Notes sur l'opéra", dans Grandeur et décadence de la ville de Mahagonny, Paris, L'Arche, [1930] 1979.

Chklovski, 1965: Viktor Chklovski, "L'art comme procédé», dans T. Todorov (dir.), Théorie de la littérature. Textes des formalistes russes réunis et traduits par Tzvetan Todorov, Paris, Seuil, 1965.

Eisenschitz, 1970: Bernard Eisenschitz, "Le Proletkult et Eisenstein", Cahiers $d u$ cinéma, $\mathrm{n}$ " 220-221, "Russie années vingt", mai-juin 1970.

Eisenstein, 1975: S. M. Eisenstein, "Le montage des attractions", en italien dans Paolo Bertetto (dir.), Ejzenstejn. FEKS. Vertov. Teoria del cinema rivoluzionario. Gli anni venti in URSS, Bologna, Feltrinelli, [1923] 1975, p. 131-135.

Eisenstein, 1986: S. M. Eisenstein, "Le montage des attractions cinématographiques", en italien dans Il Montaggio, vol. IV, tome II des Opere scelte, Venezia, Marsilio (1925), 1986, p. 227-249.

Gaudreault et Gunning, 1989: André Gaudreault et Tom Gunning, «Le cinéma des premiers temps: un défi à l'histoire du cinéma? ", dans Aumont et al., 1989, p. 49-63.

Gaudreault et Simard, 1995: André Gaudreault et Denis Simard, "L'extranéité du cinéma des premiers temps: bilan et perspectives de recherche», dans Jean A. Gili, 
M. Lagny, M. Marie et V. Pinel (dir.), Les Vingt Premières Années du cinéma français, Paris, Presses de la Sorbonne Nouvelle et AFRHC, 1995, p. 15-28.

Gaudreault, 1997: André Gaudreault, "Les vues cinématographiques selon Georges Méliès, ou: comment Mitry et Sadoul avaient peut-être raison d'avoir tort (même si c'est surtout Deslandes qu'il faut lire et relire) ", dans J. Malthête et M. Marie (dir.), Georges Méliès, l'illusionniste fin de siècle?, Paris, Presses de la Sorbonne Nouvelle, 1997, p. 111-131.

Gaudreault, 1999 : André Gaudreault, Du littéraire au filmique: Système du récit, Paris/Québec, Armand Colin/Nota Bene, [1988] 1999.

Grosoli, 1981 : Fabrizio Grosoli, Werner Herzog, Firenze, La Nuova Italia, 1981.

Gunning, 1990a : Tom Gunning, "Non-Continuity, Continuity, Discontinuity. A Theory of Genres in Early Films", dans T. Elsaesser (dir.), Early Cinema, Space, Frame, Narrative, London, BFI, 1990.

Gunning, 1990b: Tom Gunning, "The Cinema of Attractions. Early Film, Its Spectator and the Avant-Garde", dans 'T. Elsaesser (dir.), Early Cinema, Space, Frame, Narrative, London, BFI, 1990.

Gunning, 1995: Tom Gunning, "Attractions, truquages et photogénie", dans J. A. Gili, M. Lagny, M. Marie, V. Pinel (dir.), Les Vingt Premières Années du cinéma français, Paris, Presses de la Sorbonne Nouvelle/AFRHC, 1995, p. 177-193.

Gunning, 1996: Tom Gunning, "Now You See It, Now You Don't". The Temporality of Cinema of Attractions", dans R. Abel (dir.), Silent Film, London/Athlone, Rutgers University Press, 1996, p. 71-84.

Herzog, 1982: Werner Herzog, Sentieri nel ghiaccio, Milano, Guanda, 1982.

Ishagpour, 1986: Youssef Ishagpour, Cinéma contemporain: de ce côté du miroir, Paris, La Différence, 1986.

Khodoss, 1995: Florence Khodoss (dir.), Kant. Le jugement esthétique, Paris, PUF, 1995.

Fouquet, 1912: E.-L. Fouquet, "L'Attraction", dans Écho du cinéma, 28 juin, n 11, p. $1,1912$. 